\title{
Acute kidney injury strongly influences renal function after radical nephroureterectomy for upper tract urothelial carcinoma: A single-centre experience
}

\author{
Alessandro Tafuri ${ }^{1,2}$, Katia Odorizzi ${ }^{1}$, Giacomo Di Filippo ${ }^{1,3}$, Clara Cerrato ${ }^{1}$, Giulia Fassio ${ }^{1}$, \\ Emanuele Serafin ${ }^{1}$, Alessandro Princiotta ${ }^{1}$, Damiano D'Aietti ${ }^{1}$, Alessandra Gozzo ${ }^{1}$, \\ Antonio B. Porcaro ${ }^{1}$, Matteo Brunelli ${ }^{4}$, Maria Angela Cerruto ${ }^{1}$, Alessandro Antonelli ${ }^{1}$ \\ ${ }^{1}$ Department of Urology, University of Verona, Azienda Ospedaliera Universitaria Integrata Verona, Verona, Italy; \\ ${ }^{2}$ Department of Neuroscience, Imaging and Clinical Science, Physiology and Physiopathology division, "G. D’Annunzio" University, \\ Chieti, Italy; \\ ${ }^{3}$ Department of General and Hepatobiliary Surgery, University of Verona, Azienda Ospedaliera Universitaria Integrata Verona, \\ Verona, Italy; \\ ${ }^{4}$ Department of Pathology, University of Verona, Azienda Ospedaliera Universitaria Integrata Verona, Verona, Italy.
}

\begin{abstract}
Summary Objective: The aim of our study was to investigate frequency and predictors both of postoperative acute kidney injury (AKI) and renal function decline in a population of consecutive upper tract urothelial carcinoma (UTUC) patients who underwent radical nephroureterectomy (RNU).

Materials and methods: Between October 2014 and February 2020, 93 patients underwent RNU at our Institution. After considered exclusion criteria, 89 patients were selected. Perioperative clinical factors were retrospectively collected. Estimated glomerular filtration rate (eGFR) was calculated using the Chronic Kidney Disease Epidemiology Collaboration (CKDEPI) equation. We defined AKI as an increase in serum creatinine by $\geq 0.3 \mathrm{mg} / \mathrm{dl}$ or a 1.5-1.9-fold increase in serum creatinine from baseline to I post-operative day (POD). A significant renal function reduction was defined as an eGFR reduction of $40 \%$ from baseline at discharge and at last clinical evaluation. Frequency of AKI and eGFR decline was investigated. Association between perioperative clinical factors and AKI and eGFR reduction at discharged and last follow-up was studied using univariate and multivariate models.

Results: AKI was detected at I POD in 45 patients. On multivariate analysis, pre-operative eGFR was an independent predictor of AKI (OR 1.03; $p=0.042)$. Further, AKI was found to be a significant predictor of eGFR reduction $\geq 40 \%$ at discharge at univariate analysis $(O R$ 19.42; $p=0.005)$ and at multivariate analysis (OR 12.49; $p=0.02)$. In a multivariate logistic regression model post-operative AKI (OR 5.18; $p=0.033$ ), lack of ipsilateral preoperative hydronephrosis (OR 0.17; $p=0.016$ ), preoperative eGFR (OR 1.04; $p=0.047)$ and antiplatelet therapy (OR 5.14; $p=0.018$ ) were found to be independent predictors of an eGFR reduction higher than $40 \%$ at last clinical evaluation made at a median of 15 (IQR 5-30) months.

Conclusions: In our cohort, AKI was present in almost $50 \%$ of patients after RNU and it was a strong predictor of renal function decline after RNU.
\end{abstract}

KEY WORDS: Upper tract urothelial carcinoma; Radical nephroureterectomy; Acute kidney injury; Renal function reduction; Chronic kidney disease.

Submitted 26 September 2020; Accepted 15 October 2020

\section{INTRODUCTION}

Urothelial carcinoma of the upper urinary tract (UTUC) is among the ten most common cancers, is more frequent in males and diagnosis is generally done in the sixth decade $(1,2)$. Established risk factors are exposure to tobacco, arsenic, and aristolochic acid, as well as alcohol consumption (3). Some genetic polymorphisms are also associated with an increased risk of UTUC or faster disease progression that introduces variability in the inter-individual susceptibility to the risk factors previously mentioned (4). The disease has high mortality, with more than 150.000 patients dying each year worldwide (1).

Extirpative surgery with removal of kidney, entire ureter and bladder cuff - radical nephroureterectomy (RNU) - is the treatment of choice for non-metastatic high-risk UTUC. Conversely, low-risk cases (unifocal, $<2 \mathrm{~cm}$, lowgrade and superficial cancers) are amenable of kidneysparing treatments providing equal survival outcomes but better preservation of renal function (4). Despite this recommendation, a relevant rate of low-risk cases still undergo RNU for several reasons, mainly concerns on clinical understaging or challenging anatomical locations with inherent risk of tumor spillage and complications (4).

The issue of renal function impairment after RNU is generally postponed to the need for radicality, but UTUC patients are at high risk of chronic kidney disease (CKD) because of patient's age and comorbidities, smoking exposure, potential impairment of contralateral kidney due to diagnostic procedures or bilateral UTUC. Indeed, despite adjuvant chemotherapy might prolong survival (5) and reduce the risk of disease recurrence in locallyadvanced UTUC (6), nearly only $50 \%$ of patients are still eligible for platinum-based protocols, due to post-operative renal failure $(7,8)$. Finally, it should be noted that CKD might determine worse mortality due to non-cancer but also cancer-related causes, as found in patients treated for renal cell carcinoma (RCC) (9-11).

Thus, the identification of patients at risk of significant renal function decline may allow clinicians to better assess the opportunity of kidney-sparing rather than

No conflict of interest declared. 
extirpative surgery when feasible, to adopt appropriate protective strategies during the peri-operative period and to adequately schedule controls during the follow up.

The aim of our study is to investigate the prevalence of AKI and CKD, as well as the degree of renal functional impairment, in a population of patients submitted to $\mathrm{RNU}$, in order to identify the most significant predictors of these events.

\section{Materials AND MEthods}

Data were retrospectively collected in our Institutional Review Board (IRB) - approved UTUC dataset prospectively compiled since October 2014. At admission, each patient provided written informed consent for data collection and analysis.

Between October 2014 and February 2020, 93 consecutive patients underwent RNU at our Institution as primary treatment for UTUC. Patients who underwent primary neo-adjuvant chemotherapy were not considered. For the purposes of the present study 4 patients with solitary kidney were excluded, leaving 89 patients, 10 of which previously submitted to radical cystectomy.

In total 87 patients were submitted to open RNU and 2 to robot-assisted RNU.

The following data were considered: gender, age at surgery, body mass index (BMI), performance status [American Society of Anesthesiologists (ASA) classification and Eastern Cooperative Oncology Group (ECOG) score], comorbidities (presence of coronary artery disease (CAD), pulmonary disease, hypertension, hyperlipidemia, diabetes mellitus), smoking exposure, hydronephrosis, operative time, blood loss, intraoperative transfusions, pathological TNM stage ( $8^{\text {th }}$ edition) (12), grade, presence of tumor necrosis, surgical margins status, length of hospital stay.

According to an internal protocol regulating postoperative management, estimated glomerular filtration rate [eGFR, calculated by the CKD-EPI equation (13)] and blood chemistry were collected in all cases on post-operative day (POD) 1, 3 and at discharge.

Acute kidney injury was defined as an increase in serum creatinine with respect to baseline by $\geq 0.3 \mathrm{mg} / \mathrm{dl}$ or a 1.5-1.9-fold at I POD, according to the Acute Kidney Injury Network (AKIN) classification (14). According to previous reports, a renal function decline was considered significative when eGFR reduction got over $40 \%$ with respect to the baseline (15).

Based on our internal protocol for low risk patients, follow-up controls were scheduled after 3 months from surgery performing cystoscopy and blood samples. If negative, subsequent cystoscopy and urinary cytology, abdominal ultrasound, and blood samples were scheduled 9 months later and then yearly, for 5 years.

For high-risk patients, cystoscopy and urinary cytology at 3 months were performed. If negative, cystoscopy and cytology every 3 months for a period of 2 years, and every 6 months thereafter until five years, and then yearly were considered. Additionally, yearly computed tomography (CT) urography and chest CT was scheduled. However, many patients traveled to our tertiary center from far away only for surgery, and the follow-up controls were often performed elsewhere.
Table 1

Study population's characteristics $(n=89)$.

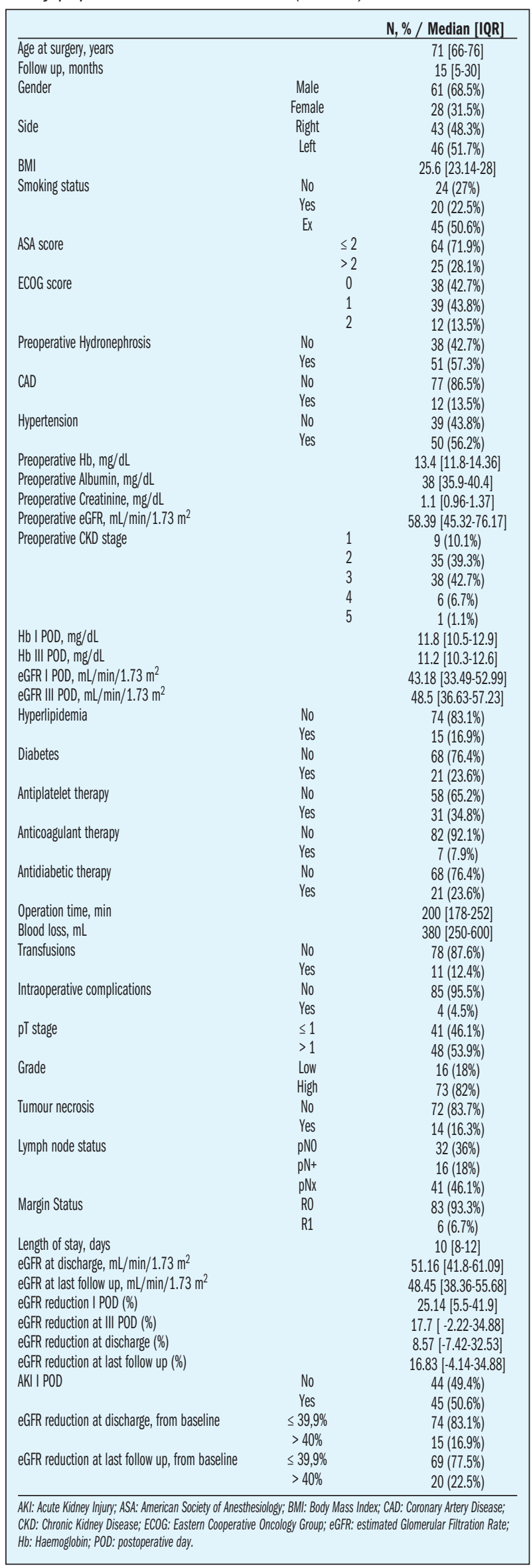


Categorical variables were expressed as frequencies and relative percentages.

Continuous variables were expressed as median and interquartile range. Patients were divided into two groups according to the presence of I POD AKI. Categorical variables were compared between groups using the $\chi^{2}$ test. Continuous variables were tested between subgroups with the independent samples t-test or MannWhitney test after testing for normality of distributions using Shapiro-Wilk test, as appropriate. Univariate logistic regression was used to identify outcomes' predictors, and Odds Ratios and 95\% confidence intervals were calculated for each significant variable. Significant variables at univariate analysis were entered into a multivariate regression model to identify independent predictors. A p value $<0.05$ was considered statistically significant. The analysis was carried out using SPSS software version 25.0 (SPSS Inc, Chicago, IL).

\section{RESULTS}

Demographics, clinical, operative and pathological data of the entire population are summarized in Table 1.

The median age of the cohort was 71 years (IQR 66-76), 61 were males, 28 females; median follow-up time was 15 months (IQR 5-30). Median preoperative $\mathrm{Hb}$ was $13.4 \mathrm{~g} / \mathrm{dL}$ (IQR 11.8-14.36) and median baseline eGFR was $58.39 \mathrm{ml} / \mathrm{min} / 1.73 \mathrm{~m}^{2}$ (IQR 45.32-76.17). 51 patients $(57.3 \%)$ had preoperative ipsilateral hydronephrosis. Histology found pTl or less in 41 patients $(46.1 \%)$ and pT2 or higher in the other 48 (53.9\%). Lymph node invasion in the pathological specimen was found in 16 (18\%) cases. Post-operative median eGFR at $1,3 \mathrm{POD}$ and at discharge were 43.18 $\mathrm{ml} / \mathrm{min} / 1.73 \mathrm{~m}^{2}$ (IQR 33.49-52.99), 48.5 $\mathrm{ml} / \mathrm{min} / 1.73 \mathrm{~m}^{2}$ (IQR 36.63-57.23) and $51.16 \mathrm{ml} / \mathrm{min} / 1.73 \mathrm{~m}^{2}$ (IQR 41.8-61.09) respectively. At the same timepoints, median relative eGFR reduction was equal to $25.14 \%, 17.7 \%$, and $8.57 \%$. At discharge and last available follow up eGFR reduction was $>40 \%$ from the baseline in 15 (16.9\%) and $20(22.5 \%)$ patients, respectively.

Overall, 45 patients $(50.6 \%)$ developed AKI after surgery. The clinicopathological characteristics of the two groups (AKI compared to non-AKI) are reported in Table 2. On univariate analysis AKI was found significantly related to preoperative $\mathrm{Hb}$ value (OR 1.44; $\mathrm{p}=0.003$ ), preoperative eGFR (OR 1.04; $\mathrm{p}=0.002)$, preoperative creatinine serum level (OR 0.23, $\mathrm{p}=0.018)$ and CKD stage $<2$ (OR 0.29, p = 0.005).

Table 2.

Clinicopathological characteristics stratified according I POD AKI.

\begin{tabular}{|c|c|c|c|c|c|}
\hline & & & $\begin{array}{l}\text { Group } 1(\mathrm{No}, \mathrm{n}=44) \\
\mathrm{N}(\%) ; \text { Median [IQR] }\end{array}$ & $\begin{array}{l}\text { Group } 1 \text { (yes, } n=45 \text { ) } \\
\mathrm{N}(\%) ; \text { Median [IQR] }\end{array}$ & $\mathrm{p}$ value \\
\hline Age at surgery, years & & & $71[66-79]$ & $72[66-76]$ & 0.663 \\
\hline Follow up, months & & & $16[5-35]$ & $15[5-29]$ & 0.660 \\
\hline \multirow[t]{2}{*}{ Gender } & Male & & $26(59.1 \%)$ & $35(77.8 \%)$ & 0.058 \\
\hline & Female & & $18(40.9 \%)$ & $10(22.2 \%)$ & \\
\hline \multirow[t]{2}{*}{ Side } & Right & & $14(31.8 \%)$ & $29(64.4 \%)$ & 0.002 \\
\hline & Left & & $30(68.2 \%)$ & $16(35.6 \%)$ & \\
\hline BMI & & & $25.63[22.99-27.65]$ & $25.6[23.4-28.8]$ & 0.159 \\
\hline \multirow[t]{3}{*}{ Smoking status } & No & & $12(27.3 \%)$ & $12(26.7 \%)$ & 0.908 \\
\hline & Yes & & $8(18.2 \%)$ & $12(26.7 \%)$ & \\
\hline & Ex & & $24(54.5 \%)$ & $21(46.7 \%)$ & \\
\hline \multirow[t]{2}{*}{ ASA score } & & $\leq 2$ & $29(65.9 \%)$ & $35(77.8 \%)$ & 0.213 \\
\hline & & $>2$ & $15(34.1 \%)$ & $10(22.2 \%)$ & \\
\hline \multirow[t]{3}{*}{ ECOG score } & & 0 & $18(40.9 \%)$ & $20(44.4 \%)$ & 0.355 \\
\hline & & 1 & $22(50 \%)$ & $17(37.8 \%)$ & \\
\hline & & 2 & $4(9.1 \%)$ & $8(17.8 \%)$ & \\
\hline \multirow[t]{2}{*}{ Preoperative Hydronephrosis } & No & & $15(34.1 \%)$ & $23(51.1 \%)$ & 0.105 \\
\hline & Yes & & $29(65.9 \%)$ & $22(48.9 \%)$ & \\
\hline \multirow[t]{2}{*}{ CAD } & No & & $41(93.2 \%)$ & $36(80 \%)$ & 0.069 \\
\hline & Yes & & $3(6.8 \%)$ & $9(20 \%)$ & \\
\hline \multirow[t]{2}{*}{ Hypertension } & No & & $22(50 \%)$ & $17(37.8 \%)$ & 0.245 \\
\hline & Yes & & $22(50 \%)$ & $28(62.2 \%)$ & \\
\hline Preoperative Hb, mg/dL & & & 12.65 [11.05-13.9] & 13.9 [13-15.2] & 0.001 \\
\hline Preoperative Albumin, mg/dL & & & $37.7[35.55-40.35]$ & $38[36.3-40.9]$ & 0.042 \\
\hline Preoperative Creatinine, $\mathrm{mg} / \mathrm{dL}$ & & & $1.21[0.98-1.68]$ & $1.05[0.91-1.24]$ & 0.001 \\
\hline Preoperative eGFR, $\mathrm{mL} / \mathrm{min} / 1.73 \mathrm{~m}^{2}$ & & & $51.34[40.97-65.89]$ & $69.37[53.46-80.37]$ & 0.001 \\
\hline \multirow[t]{5}{*}{ Preoperative CKD stage } & & 1 & $3(6.8 \%)$ & $6(13.3 \%)$ & 0.044 \\
\hline & & 2 & $12(27.3 \%)$ & $23(51.1 \%)$ & \\
\hline & & 3 & $23(52.3 \%)$ & $15(33.3 \%)$ & \\
\hline & & 4 & $5(11.4 \%)$ & $1(2.2 \%)$ & \\
\hline & & 5 & $1(2.3 \%)$ & $0(0 \%)$ & \\
\hline $\mathrm{Hb} \mid \mathrm{POD}, \mathrm{mg} / \mathrm{dL}$ & & & $11.6[10.35-12.6]$ & $12[10.5-13.4]$ & 0.007 \\
\hline $\mathrm{Hb}$ III POD, mg/dL & & & $11.45[10.5-12.5]$ & $10.9[10.1-12.7]$ & 0.003 \\
\hline eGFR I POD, mL/min/1.73 m² & & & $51.13[41.67-62]$ & 38.44 [31.66-45.42] & 0.001 \\
\hline eGFR III POD, mL/min $/ 1.73 \mathrm{~m}^{2}$ & & & $51.01[41.31-68.16]$ & $45.42[36.31-54.13]$ & 0.001 \\
\hline \multirow[t]{2}{*}{ Hyperlipidemia } & No & & $36(81.8 \%)$ & $38(84.4 \%)$ & 0.741 \\
\hline & Yes & & $8(18.2 \%)$ & $7(15.6 \%)$ & \\
\hline Diabetes & No & & $32(72.7 \%)$ & $36(80 \%)$ & 0.419 \\
\hline & Yes & & $12(27.3 \%)$ & $9(20 \%)$ & \\
\hline Antiplatelet therapy & No & & $33(75 \%)$ & $25(55.6 \%)$ & 0.054 \\
\hline & Yes & & $11(25 \%)$ & $20(44.4 \%)$ & \\
\hline Anticoagulant therapy & No & & $40(90.9 \%)$ & $42(93.3 \%)$ & 0.671 \\
\hline & Yes & & $4(9.1 \%)$ & $3(6.7 \%)$ & \\
\hline Antidiabetic therapy & No & & $32(72.7 \%)$ & $36(80 \%)$ & 0.419 \\
\hline & Yes & & $12(27.3 \%)$ & $9(20 \%)$ & \\
\hline Operation time, min & & & 200 [161-251] & 200 [180-252] & 0.928 \\
\hline Blood loss, mL & & & $400[275-600]$ & $365[250-650]$ & 0.388 \\
\hline Transfusions & No & & $39(88.6 \%)$ & $39(86.7 \%)$ & 0.778 \\
\hline & Yes & & $5(11.4 \%)$ & $6(13.3 \%)$ & \\
\hline Intraoperative complications & No & & $44(100 \%)$ & $41(91.1 \%)$ & 0.043 \\
\hline & Yes & & $0(0 \%)$ & $4(8.9 \%)$ & \\
\hline pT stage & $\leq 1$ & & $15(34.1 \%)$ & $26(57.8 \%)$ & 0.025 \\
\hline & $>1$ & & $29(65.9 \%)$ & $19(42.2 \%)$ & \\
\hline Grade & Low & & $7(15.9 \%)$ & $9(20 \%)$ & 0.615 \\
\hline & High & & $37(84.1 \%)$ & $36(80 \%)$ & \\
\hline Tumour necrosis & No & & $35(83.3 \%)$ & $37(84.1 \%)$ & 0.924 \\
\hline & Yes & & $7(16.7 \%)$ & $7(15.9 \%)$ & \\
\hline Lymph node status & pNO & & $17(38.6 \%)$ & $15(33.3 \%)$ & 0.615 \\
\hline & $\mathrm{pN+}$ & & $9(20.5 \%)$ & $7(15.6 \%)$ & \\
\hline & $\mathrm{pNx}$ & & $18(40.9 \%)$ & $23(51.1 \%)$ & \\
\hline Margin Status & RO & & $38(86.4 \%)$ & $45(100 \%)$ & 0.010 \\
\hline & R1 & & $6(13.6 \%)$ & $0(0 \%)$ & \\
\hline Length of stay, days & & & 10 [8-11] & $10[9-13]$ & 0.993 \\
\hline eGFR at discharge, $\mathrm{mL} / \mathrm{min} / 1.73 \mathrm{~m}^{2}$ & & & 53.7 [44.49-64.32] & $46.98[41.35-54.67]$ & 0.004 \\
\hline eGFR at last follow up, $\mathrm{mL} / \mathrm{min} / 1.73 \mathrm{~m}^{2}$ & & & $50.43[39.41-59.84]$ & $46.98[38.32-54.67]$ & 0.001 \\
\hline eGFR reduction I POD (\%) & & & $5.44[-7.94-12.75]$ & $41.9[35.50-48.89]$ & 0.001 \\
\hline eGFR reduction at III POD (\%) & & & $-0.35[-18.41-13.48]$ & $34.83[22.90-42.15]$ & 0.001 \\
\hline eGFR reduction at discharge (\%) & & & $-3.16[-16.63-4.68]$ & $30.69[10.66-43.09]$ & 0.001 \\
\hline eGFR reduction at last follow up (\%) & & & $-1.31[-10.62-17]$ & $33.17[13.36-44.92]$ & 0.001 \\
\hline eGFR reduction at discharge, from baseline & $\leq 39,9 \%$ & & $43(97.7 \%)$ & $31(68.9 \%)$ & 0.001 \\
\hline & $>40 \%$ & & $1(2.3 \%)$ & $14(31.1 \%)$ & \\
\hline eGFR reduction at last follow up, from baseline & $\leq 39,9 \%$ & & $41(93.2 \%)$ & $28(62.2 \%)$ & 0.001 \\
\hline & $>40 \%$ & & $3(6.8 \%)$ & $17(37.8 \%)$ & \\
\hline
\end{tabular}


On multivariate analysis, preoperative eGFR was the only independent predictor to the occurrence of AKI (OR 1.03; $\mathrm{p}=0.042)$ (Table 3).

On univariate analysis AKI (OR 19.42; $\mathrm{p}=0.005)$, preoperative eGFR (OR 1.05; $\mathrm{p}=0.004)$, preoperative $\mathrm{Hb}$ (OR 1.43; $\mathrm{p}=0.036)$, the lack of ipsilateral hydronephrosis (OR 0.30; $p=0.047$ ), III POD creatinine serum level (OR 3.00; p = 0.018), I POD Hb (OR 1.57, p = 0.019) were predictors of eGFR reduction $>40 \%$ at discharge.

On multivariate analysis only AKI retained its significance (OR 12.49; $\mathrm{p}=0.02$ ) (Table 4).

Among several factors predicting eGFR reduction > 40\% at last follow-up on univariate analysis, AKI (OR 5.18, $p=0.033)$, preoperative eGFR (OR 1.04, $p=0.047)$, the lack of ipsilateral hydronephrosis (OR 0.17; $\mathrm{p}=0.016)$, and antiplatelet therapy (OR 5.14; $\mathrm{p}=0.018$ ) were found significantly associated to the outcome also on multivariate analysis (Table 5).

\section{Table 3.}

Logistic regression analysis for I POD AKI predictors assessment.

\begin{tabular}{|l|ccc|ccc|}
\hline & \multicolumn{3}{|c|}{ Univariate } & \multicolumn{3}{c|}{ Multivariate } \\
\hline & $\mathbf{O R}$ & $\mathbf{9 5 \%} \mathbf{C I}$ & $\mathbf{p}$ value & $\mathbf{O R}$ & $\mathbf{9 5 \%} \mathbf{~ C l}$ & $\mathbf{p}$ value \\
\hline Preoperative CKD stage & 0.285 & $0.12-0.68$ & 0.005 & & & \\
Preoperative creatinine & 0.234 & $0.07-0.78$ & 0.018 & & & \\
Preoperative eGFR & 1.038 & $1.01-1.06$ & 0.002 & 1.027 & $1.00-1.05$ & 0.042 \\
Preoperative Hb & 1.441 & $1.13-1.83$ & 0.003 & 1.295 & $0.99-1.68$ & 0.051 \\
\hline AKI: Acute Kidney Injury; CKD: Chronic Kidney Disease; eGFR: estimated Glomerular Filtration Rate; \\
Hb: Haemoglobin; POD: postoperative day.
\end{tabular}

Table 4.

Logistic regression analysis for the assessment of eGFR reduction $>40 \%$ from baseline to discharge.

\begin{tabular}{|l|ccc|ccc|}
\hline \multicolumn{3}{|c}{ Univariate } & \multicolumn{3}{c|}{ Multivariate } \\
\hline & OR & $\mathbf{9 5 \%}$ Cl & p value & OR & $\mathbf{9 5 \%}$ Cl & p value \\
\hline Preoperative Hb & 1.43 & $1.02-2$ & 0.036 & 1.091 & $0.76-1.57$ & 0.636 \\
Hb I POD & 1.567 & $1.08-2.28$ & 0.019 & & & \\
Preoperative eGFR & 1.053 & $1.02-1.09$ & 0.004 & 1.036 & $0.99-1.08$ & 0.108 \\
AKI I POD & 19.419 & $2.42-155.54$ & 0.005 & 12.491 & $1.48-105.40$ & 0.02 \\
Creatinine III POD & 3.004 & $1.21-7.45$ & 0.018 & & & \\
Preoperative hydronephrosis & 0.304 & $0.09-0.98$ & 0.047 & 0.519 & $0.13-2.02$ & 0.344 \\
\hline AKl: Acute Kidney Injury; CKD: Chronic Kidney Disease; eGFR: estimated Glomerular Filtration Rate; & \\
Hb: Haemoglobin; POD: postoperative day.
\end{tabular}

Table 5.

Logistic regression analysis for the assessment of eGFR reduction $>40 \%$ from baseline to the last follow-up.

\begin{tabular}{|c|c|c|c|c|c|c|}
\hline & \multicolumn{3}{|c|}{ Univariate } & \multicolumn{3}{|c|}{ Multivariate } \\
\hline & OR & $95 \% \mathrm{Cl}$ & p value & OR & $95 \% \mathrm{Cl}$ & p value \\
\hline Preoperative Hb & 1.359 & $1.02-1.81$ & 0.037 & 1.085 & $0.75-1.57$ & 0.663 \\
\hline $\mathrm{Hb} \mid \mathrm{POD}$ & 1.598 & $1.13-2.26$ & 0.008 & & & \\
\hline Hb III POD & 1.768 & $1.21-2.58$ & 0.003 & & & \\
\hline Preoperative eGFR & 1.055 & $1.02-1.09$ & 0.001 & 1.043 & $1-1.09$ & 0.047 \\
\hline AKI I POD & 8.298 & $2.22-31$ & 0.002 & 5.183 & $1.14-23.54$ & 0.033 \\
\hline Creatinine III POD & 67.524 & 8.45-539.64 & 0.0001 & & & \\
\hline Creatinine at discharge & 72.359 & $9.87-530.65$ & 0.0001 & & & \\
\hline Preoperative hydronephrosis & 0.167 & $0.05-0.52$ & 0.002 & 0.172 & $1.14-23.55$ & 0.016 \\
\hline Antiplatelet therapy & 3.947 & $1.4-11.16$ & 0.01 & 5.139 & $1.32-19.93$ & 0.018 \\
\hline
\end{tabular}

\section{Discussion}

The present study shows that patients with UTUC candidate to RNU have baseline poor renal function, with median eGFR values close to $60 \mathrm{ml} / \mathrm{min}$, and post-operatively suffer from further relevant decline given that an impairment exceeding $40 \%$ of baseline function was noted in $22.5 \%$ of cases at a median follow-up of 15 months.

The factors associated with worse functional outcome were preoperative eGFR, lack of ipsilateral hydronephrosis, antiaggregating therapy and the presence of post-operative AKI, which represent the strongest predictor of CKD after RNU in the present cohort.

Definitely these patients have major determinants of baseline impaired function and relevant risks to develop CKD, with inherent effects on non-cancer (11), but also cancer-related survival outcomes, firstly concerning the access to platinum-based chemotherapy regimens that showed significantly improved disease-free survival in locally advanced UTUC (6).

Although renal function preservation in UTUC represents a major issue it has been poorly investigated, with sparse reports in the literature. The main reason of this is that UTUC is often featured by aggressiveness and multifocality, so that extirpative treatment is commonly privileged, except for very selected low risk cases for whose kidney-sparing approaches might be preferred $(16,17)$. In 2006, Meyer et al. retrospectively analyzed 131 RNU patients reporting a $18 \%$ deterioration in eGFR after a median follow-up of 5 years. Such deterioration was found to be greater in patients with older age, and comorbidities as diabetes mellitus, hypertension, pre-existing renal impairment and analgesic nephropathy (18).

In a multicentric retrospective study evaluating 388 patient who underwent RNU for UTUC, Kaag MG et al. showed a mean $24 \%$ of eGFR decrease after surgery. They also reported that eligibility to platinum-based chemotherapy decreased from $49 \%$ before surgery to $19 \%$ post-surgery using a cut-off of $60 \mathrm{~mL} / \mathrm{min} / 1.73 \mathrm{~m}^{2}$, and from $80 \%$ to $55 \%$ using a cut-off of $45 \mathrm{~mL} / \mathrm{min} / 1.73 \mathrm{~m}^{2}$ (19).

Kaag M. and his group identified age and preoperative eGFR as a predictors of renal function decline after RNU (20). They retrospectively enrolled 374 RNU patients and assessed early (1-5 months) and late (> 5 months) eGFR after surgery: multivariable analysis identified preoperative eGFR lower than $60 \mathrm{~mL} / \mathrm{min} / 1.73 \mathrm{~m}^{2}$ and age $>70$ years as preoperative predictors of clinically relevant eGFR loss after RNU, considering clinically relevant a loss of renal function compromising the possibility of chemotherapy recruitment.

Shao et al. recently reported that among 242 RNU cases, $42.1 \%$ was eligible to cisplatin-based therapy prior to RNU whereas, following surgery, only $15.2 \%$ remained eligible, because of the worsening of renal function (8). In the present study, we also investigated the role of postoperative AKI, finding that a half of patients experienced this event. Interestingly, preoperative hemoglobin and preoperative eGFR predicted AKI, but only preoperative eGFR remained an independent predictor of AKI on multivariate analysis.

The prevalence of AKI after RNU was previously unreported and resembles the data after radical nephrectomy for RCC (15). The most relevant finding of our analysis 
is that AKI affects long-term renal function impairment, indicating that any effort should be done to prevent AKI, especially in patients at risk.

Identification of such patients would allow to optimize the perioperative management in order to reduce the incidence of AKI after surgery and its consequences. A dedicated pre-, intra- and postoperative management with avoidance of potentially nephrotoxic agents, close monitoring of serum creatinine and urine output (remembering that urine output and serum creatinine are changing very late during the development of AKI), optimization of volume status and hemodynamic parameters and use of alternatives to radio contrast agents, represent the best AKI preventative measure to adopt in perioperative time (2123). Also, anesthesiologist may contribute to renal damage prevention avoiding the reduction of renal blood and renal hypoxia, and preventing hypotension during surgery (21). The median percentage eGFR reduction we sought was $8.6 \%$ at discharge, $16.8 \%$ at last follow-up after a median of 15 months from surgery. The rate of patients experiencing an eGFR decline $\geq 40 \%$ at last follow-up was $22.5 \%$. The factors independently associated to this event were post-operative AKI, the lack of preoperative hydronephrosis, preoperative hemoglobin, and antiplatelet therapy. The interpretation of these findings is that already established contralateral hypertrophy due to hydronephrosis of the affected urinary tract, as well as better post-operative course, with less blood loss and without AKI, facilitate the compensatory role of the remnant solitary kidney. Additionally, antiplatelet drugs represent a risk factors for renal function impairing, especially after RNU. As we already mentioned, these patients should be properly managed pre-operatively and accurately followed after surgery. After nephrectomy UTUC patients showed larger eGFR reduction than those with parenchymal tumors, reasonably because the latter are generally younger and with less comorbidities. However, there are some intrinsic differences between these two conditions still to be investigated. Tae et al. indeed investigated by matched-pair comparison 554 patients who underwent nephrectomy for UTUC $(\mathrm{n}=277)$ or parenchymal tumor $(\mathrm{n}=277)$, balanced in terms of age, BMI, baseline eGFR and comorbidities. A significant larger decline in postoperative eGFR was found in UTUC cases $73.3 \%$ vs. $66.1 \%$, $\mathrm{p}=0.039)$ and multivariate analysis showed that the indication to nephrectomy due to UTUC (OR 1.84; $\mathrm{p}=$ 0.006) was an independent predictor of postoperative impaired renal function (24). Lee et al. showed similar findings in 616 patient who underwent nephrectomy for renal cancer ( $n=319)$, or UTUC $(n=297)$, with the latter older and more comorbid. The authors reported that UTUC patients had an increased risk of serum creatinine doubling and need for dialysis after radical nephrectomy, and the predictor of these unfavorable outcomes were old age, diabetes, low baseline eGFR and indication to nephrectomy due to UTUC (25).

Other tools have been proposed for identifying and monitoring patients at risk of renal failure. Brardi et al. investigated the role of doppler ultrasound derived renal resistive index (RRI) in a CKD population in the monitoring of renal function after a therapeutic and dietetic intervention to ameliorate the renal impairment.
The authors found that RRI was a key parameter in monitoring patients with CKD and a helpful tool to drive clinical efforts to contrast renal function decline (26).

The same team recently found that variation in time of eGFR positively correlates to sonographic measurements of average right and left kidney diameters and percentage variations of right and left renal cortical thickness in a population of 80 adult patients with various degrees of chronic kidney disease after received of a therapeutic and dietetic intervention to improve renal function. Patients were not dialysis-dependent, they did not undergo renal surgery, nor they were affected by any of the pathological conditions that can increase kidney size (27). According to these pieces of evidence, renal ultrasound derived parameters together with clinical factors could represent a useful tool for evaluating patients before RNU and subsequent follow up.

The present study is a retrospective evaluation of a small population. However, our results are innovative. We showed that AKI at I POD is a strong predictor of renal function decline in patients who underwent RNU for UTUC who might need an adjuvant platinum-based chemotherapy. Identifying patients at high-risk of renal function decline has a pivotal role to provide a correct peri-operative management. A tailored pre-operative management and surgical procedure should be provided to patients at high risk of developing AKI. Additionally, when UTUC patients are counseled before treatment, the risk of renal function decline should be extensively explained. Further higher-level studies are needed to confirm our results.

\section{Conclusions}

In our cohort, almost 50\% of patients developed AKI after RNU. Acute kidney injury was a strong predictor of renal function decline after radical nephroureterectomy at discharged and a 15 months follow-up. Identifying patients at high-risk of renal function decline is essential to provide a correct peri-operative management.

\section{REFERENCES}

1. Siegel RL, Miller KD, Jemal A. Cancer statistics. CA Cancer J Clin. 2019; 69:7-34.

2. Horstmann M, Witthuhn R, Falk M, Stenzl A. Gender-specific differences in bladder cancer: a retrospective analysis. Gend Med. 2008; 5:385-94.

3. Colin P, Koenig P, Ouzzane A, et al. Environmental factors involved in carcinogenesis of urothelial cell carcinomas of the upper urinary tract. BJU international, 2009; 104:1436-1440.

4. Rouprêt M, Babjuk M, Burger M, et al. European Association of Urology Guidelines on Upper Urinary Tract Urothelial Carcinoma: 2020 Update. Eur Urol. 2020:S0302-2838(20)30427-9.

5. Bamias A, Moulopoulos LA, Koutras A, et al. The combination of gemcitabine and carboplatin as first-line treatment in patients with advanced urothelial carcinoma. A Phase II study of the Hellenic Cooperative Oncology Group. Cancer. 2006; 106:297-303.

6. Birtle A, Johnson M, Chester J, et al. Adjuvant chemotherapy in upper tract urothelial carcinoma (the POUT trial): a phase 3, openlabel, randomised controlled trial. Lancet. 2020; 395:1268-1277. 
7. Galsky MD, Hahn NM, Rosenberg J, et al. Treatment of patients with metastatic urothelial cancer "unfit" for cisplatin-based chemotherapy. J Clin Oncol. 2011; 29:p 2432-2438.

8. Shao IH, Lin YH, Hou CP, et al. Risk factors associated with ineligibility of adjuvant cisplatin-based chemotherapy after nephroureterectomy. Drug Des Devel Ther. 2014; 8:1985-90.

9. Antonelli A, Minervini A, Sandri M, et al. Below safety limits, every unit of glomerular filtration rate counts: assessing the relationship between renal function and cancer-specific mortality in renal cell carcinoma. Eur Urol. 2018; 74:661-667.

10. Antonelli A, Palumbo C, Sandri M, et al. Renal function impairment below safety limits correlates with cancer-specific mortality in localized renal cell carcinoma: results from a single-center study. Clin Genitourin Cancer. 2020; 18:e360-e367.

11. Go AS, Chertow GM, Fan D, et al. Chronic kidney disease and the risks of death, cardiovascular events, and hospitalization. New Engl J Med. 2004; 351:1296-1305.

12. Brierley JD, Gospodarowicz MK, Wittekind C. TNM classification of malignant tumours. 2017: John Wiley \& Sons.

13. Levey AS, Stevens LA, Schmid CH, et al., A new equation to estimate glomerular filtration rate. Ann Int Med. 2009; 150:604-612.

14. Bellomo R, Ronco C, Kellum JA, et al. Acute Dialysis Quality Initiative workgroup. Acute renal failure - definition, outcome measures, animal models, fluid therapy and information technology needs: the Second International Consensus Conference of the Acute Dialysis Quality Initiative (ADQI) Group. Crit Care 8:R204-12.

15. Garofalo C, Liberti ME, Russo D, et al. Effect of post-nephrectomy acute kidney injury on renal outcome: a retrospective long-term study. World J Urol. 2018; 36:59-63.

16. Yakoubi R, Colin P, Seisen T, et al. Radical nephroureterectomy versus endoscopic procedures for the treatment of localised upper tract urothelial carcinoma: a meta-analysis and a systematic review of current evidence from comparative studies. Eur J Surg Oncol. 2014; 40:1629-34

17. Fang $D$, Seisen $T$, Yang $K$, et al. A systematic review and metaanalysis of oncological and renal function outcomes obtained after segmental ureterectomy versus radical nephroureterectomy for upper tract urothelial carcinoma. Eur J Surg Oncol. 2016; 42:16251635

18. Meyer JP, Delves GH, Sullivan ME, et al. The effect of nephroureterectomy on glomerular filtration rate. BJU international. 2006; 98: 845-848.

19. Kaag MG, O'Malley RL, O'Malley $P$, et al. Changes in renal function following nephroureterectomy may affect the use of perioperative chemotherapy. Eur Urol. 2010; 58:581-587.

20. Kaag M,Trost $L$, Thompson $R H$, et al. Preoperative predictors of renal function decline after radical nephroureterectomy for upper tract urothelial carcinoma. BJU international. 2014; 114:674-679.

21. Zarbock A, Koyner JL, Hoste EAJ, et al. Update on perioperative acute kidney injury. Anesth Analg, 2018; 127:1236-1245.

22. KDIGO clinical practice guideline for acute kidney injury. Kidney Int Suppl. 2012; 2:1-138.

23. Bell S, Prowle J. Postoperative AKI-Prevention Is better than cure? J Am Soc Nephrol. 2019; 30:4-6.

24. Tae BS, Ku JH, Kwak C, et al. Comparison of renal function after radical surgery for upper tract urothelial carcinoma versus renal cell carcinoma: propensity score matching. Urologia Internationalis. 2018; 101:400-408.
25. Lee, KH, Chen YT, Chung HJ, et al. Kidney disease progression in patients of upper tract urothelial carcinoma following unilateral radical nephroureterectomy. Renal failure. 2016; 38:77-83.

26. Brardi S, Cevenini G, Giovannelli V, Romano G. Longitudinal prospective observational type study about determinants of renal resistive index variations in chronic renal failure patients treated with conventional medical and dietetic therapy. Arch Ital Urol Androl. 2017; 89:305-309.

27. Brardi S, Cevenini G. Time changes of renal dimensions and variations of glomerular filtration rate in chronic kidney disease patients. Arch Ital Urol Androl. 2020; 92:21-24.

\section{Correspondence}

Alessandro Tafuri, MD aleta@hotmail.it

Katia Odorizzi, MD

katia.odorizzi@virgilio.it

Giacomo Di Filippo, MD

giacomo.difilippo90@gmail.com

Clara Cerrato, MD

clara.cerrato01@gmail.com

Giulia Fassio, MD

fassio.giulia@gmail.com

Emanuele Serafin, MD

serafin.mnl@gmail.com

Alessandro Princiotta, MD

alessandroprinciotta0@gmail.com

Damiano D'Aietti, MD

damiano.daietti@gmail.com

Alessandra Gozzo, MD

la.ale.gozzo@gmail.com

Antonio B. Porcaro, MD

antoniobenito.porcaro@aovr.veneto.it

Maria Angela Cerruto, MD

mariaangela.cerruto@univr.it

Alessandro Antonelli, MD (Corresponding Author)

alessandro.antonelli@univr.it

Department of Urology, University of Verona, Azienda Ospedaliera

Universitaria Integrata Verona, Piazzale Stefani 1, 37126, Verona (Italy)

Matteo Brunelli, MD

matteo.brunelli@univr.it

Department of Pathology, University of Verona,

Azienda Ospedaliera Universitaria Integrata Verona, Verona (Italy) 